exponent of biological psychiatry for many years. In this book he brings together the results of all the recent research on the biological factors in psychiatry. Unlike the "dynamic" psychiatrist he is prepared to admit that there are other approaches to psychiatric problems apart from his own. In the first chapter, which is written in collaboration with Dr Norman Kreitman, there is a well balanced account of the genetic and psychosocial factors in the causation of schizophrenia, which should be read by all over-enthusiastic "organic" and "dynamic" psychiatrists. The biochemical theories and findings in schizophrenia aro adequately dealt with in the second chapter, while the third chapter, written in conjunction with Dr Alec Coppen, deals with the recent work on the biochemistry of the affective psychoses. Smythies then discusses his investigations of hallucinogenic drugs in animals and after this goes on to speculate about the brain mechanisms responsible for human behaviour. The book ends with an excellent account of the theoretical basis of psychoanalysis and a discussion of the way in which this theory can be reconciled with experimental findings.

This book is most welcome because it brings together a large amount of material on the biological basis of psychiatry which is at present scattered through the literature. The publishers and the author are to be congratulated on producing such an up to date book, which refers to so much recent work. The material in this book is in fact no more out of date than the average article in a current technical journal. This excellent survey of an important aspect of psychiatry should be read by all psychiatrists and clinical psychologists.

While Smythies's book is on the whole derivative and most of it will be out of date within a few years, Kringlen's monograph, based as it is on original work, will be a standard work for many years to come. The author begins by reviewing the previous twin studies of the functional psychoses and then presents his own work in this field. His conclusions are: "The difference in concordance rates for monozygotic and dizygotic twins with respect to schizophrenia is statistically significant, thus supporting a genetic factor in the etiology of schizophrenia, but the genetic factor seems to be weaker than it is usually considered to be".

An important finding which Kringlen stresses is that a number of schizophrenic probands have psychiatrically abnormal twins who are not schizophrenic, and in the past many investigators have used rather loose diagnostic criteria for schizophrenia and inflated the concordance rate. Kringlen's findings are supported by those of Heston, who found that, apart from an excess of schizophrenia, there was also an excoss of abnormal personalitios and neuroses in the children of schizophrenic mothers. As Kringlen puts it: "In other words, the clinical pictures presented by individuals with the same hereditary equipment as the schizophrenic embrace a graduated serics of disorders and personality patterns that ranges from a duplication of the psychosis to nourosis and clinical normaley".

This raises a difficult problem for the average European psychiatrist who has been trained to regard schizophrenia as an illness. It may be that the abnormal neurophysiological processes which cause the functional psychoses do not each produce one clinical picture, so that the same psychiatric "illness" may be the result of many different discasc processes. Kringlen does not accept this view, but feels that the best way of accounting for the facts is to assunne that there is a polygenic mode of inheritance in schizophrenia.

Apart from the problems of concordance, Kringlen discusses the environmental and social factors which may play a part in the causation of schizophrenia. The result is a well balanced book which considers all aspects of the causation of schizophrenia.

Unfortunately, no firm conclusions can be drawn about the inheritance of the manic-depressive psychoses, because the number of monozygotic twin probands with these illnesses reported in the literature and found by the author is relatively small.

One minor point of criticism is that there is not an adequate discussion of the geneties of the schizophreniform or atypical psychoses and the work of Leonhard, and Elsässer and Colmant, in this field is not mentioned. This is, however, a minor blemish in an excellent monograph which must be read by everyone interested in the functional psychoses.

Frank Fish

\section{SCIENCE AND THERAPEUTICS}

\section{Modern Trends in Pharmacology and Therapeutics 1}

Edited by W. F. M. Fulton. Pp. $\mathrm{x}+372$. (Butterworth : London, 1967.) 85s.

THF stated editorial objective of this new series is to provide postgraduate students of internal medicine and clinicians with the scientific basis of present day therapeutics. The form of presentation used recognizes the problems facing an individual author when expected to cover so broad a field as that of therapeuties; the publishers have, therefore, wisely decided to invite subject specialists to describe the recent developments that have occurred in their own area of specialization. Nevertheless, the primary emphasis on pharmacological and physio. logical mechanism of action has been well maintained within each chapter and the texts are also sufficiently well referonced to direct its readers to moro detailed literature should the need arise.

The selected fields of therapeutic interest are broad ranging and apart from a complete omission of psycho. pharmacological agents, which are presumably to be the subject of future volumes, reflect the chief areas of current clinical interest. The first chapter contains a general discussion of the factors affecting drug action and usefully collects together in a single chapter important features such as membrane permeability, specialized membrane barriers, excretion, binding and storage depots. In addition, much useful information on the nature of drug interactions with respect to antagonism, biotransformation, physico-chemical aspects of recoptor attachment and structure action relationship are gathered together in this chapter. The remaining twelve chapters include two on aspects of cardiovascular therapy, one of which is concerned with cardiotonic and anti-arrhythmic agents and the other on drugs which modify blood pressure. Three fields of chemotherapy are reviewed, with one chapter on antiviral drugs, another on antibacterial agents and the third on tumour growth inhibiting substances. The use of oxygen therapy in conditions of respiratory failure and cardiovascular collapse and the status of hyperbaric oxygen in clinical treatment are also adequately discussed. In addition, there are individual chapters on agents which are used to modify fertility, diuretic drugs, blood fibrinolytic agents and the use of magnesium salts to correct deficient states arising from metabolic imbalances. One other interesting inclusion is a discussion on the control of hyperlipidaemia using dietary and therapeutic methods. The pharmacological principles are all well cxpounded and their relationships to the therapcutic problems are usually clear and pertinent.

The volume successfully attains its objective of linking the developments in pharmacology to the advances currently occurring in therapeutics and as a result other interested groups such as clinical pharmacologists and pharmacists are also likely to benefit from the provision of such a series. If the series continues with the promise shown in the first volume, one may venture favourably as to the ultimate success of the entire series.

G. D. H. LeaciI 